\title{
ARTICLE
}

\section{Generation and evaluation of gamma-ray buildup factors prepared as the standard data in the Atomic Energy Society of Japan}

\author{
Akinao Shimizu $^{\mathrm{a}^{*}}$, Yukio Sakamoto ${ }^{\mathrm{b}}$ and Yoshihiro Hirao ${ }^{\mathrm{c}}$ \\ ${ }^{a}$ NPO Radiation Dose Analysis and Evaluation, 4-13-14, Kitamachi, Kokubunji-shi, Tokyo 185-0001, Japan; ${ }^{b}$ Japan Atomic Energy \\ Agency, 2-4 Shirakata, Tokai-mura, Naka-gun, Ibaraki-ken 319-1195, Japan; ${ }^{c}$ National Maritime Research Institute, 6-38-1, \\ Shinkawa, Mitaka-shi, Tokyo 181-0004, Japan
}

\begin{abstract}
A data set of gamma-ray buildup factors for point isotropic sources has been generated by the method of invariant embedding. Specific features of the data set as compared with the ANSI/ANS-6.4.3 standard published by the American Nuclear Society are 1) the data corresponding to effective dose with AP and ISO irradiation geometries, and ambient dose equivalents are added, 2) the distance from the source is extended up to 100 mean free paths (mfp) from $40 \mathrm{mfp}$ in the ANS data, 3) the method of invariant embedding and the photon cross section PHOTX are used consistently for all materials, and 4) treatment of bremsstrahlung is improved by using the bremsstrahlung production data calculated by the EGS4 code. The accuracy of the transport calculations for exposure buildup factors by the IE method was evaluated quantitatively by works including 1) comparison of buildup factors with those obtained by the moments method in the ANS data, 2) comparison of the buildup factors with those obtained by the EGS4 code, and 3) self evaluation of influence of mesh width in space, energy and angle in the numerical solution of transport equation by the IE method. It is concluded that the magnitude of error in the present data is estimated about $10 \%$ or less up to $40 \mathrm{mfp}$.
\end{abstract}

Keywords: standard data; gamma-ray buildup factors; point isotropic sources; invariant embedding method

\section{Introduction}

A data set of gamma-ray buildup factors for point isotropic sources in infinite homogeneous media has been generated by the method of invariant embedding (IE method). The data set has been evaluated by the working group on gamma-ray buildup factors of the subcommittee on radiation shielding, and prepared as the standard data in the Atomic Energy Society of Japan. In the following sections, the IE method and quantitative estimations of error in numerical solutions will be described in more detail. The improved treatment of bremsstrahlung is given by Sakamoto et. al. [1]

\section{Extension from ANS data}

Specific features of the data set as extended from the ANSI/ANS-6.4.3 standard published by the American Nuclear Society (ANS data) [2] are 1) rubidium and bismuth are added to 26 engineering materials in the ANS data, 2) the data corresponding to effective dose with AP and ISO irradiation geometries, and ambient dose equivalents are added to those corresponding to exposure and energy absorption in the ANS data, 3) the distance from the source is extended up to 100 mean free paths (mfp) from $40 \mathrm{mfp}$ in the ANS data, 4) the IE method and the photon cross section database PHOTX [3] are used consistently for all materials and source energies, and 5) treatment of bremsstrahlung is improved by using the bremsstrahlung production data calculated by the EGS4 code, and 6) quantitative estimations of error in numerical solutions obtained by the IE method are made.

\section{Invariant embedding method}

\subsection{Introduction}

The method of invariant embedding (IE method) was first introduced by Ambaruzumian (1934) and Chndrasekhar (1950) in the field of astrophysics to solve the problem of diffuse reflection of light by a stellar atmosphere. The method was further generalized by Bellman and Kalaba (1956) in the field of mathematical physics. Bellman named the method as "invariant embedding". The Method was further extended by Shimizu and Mizuta (1966) to transport problems of gamma-ray dependent with energy and angle.

*Corresponding author. Email: ashi1041@fancy.ocn.ne.jp 


\subsection{Outline of the IE method}

\subsubsection{Calculation of reflection function}

A reflection function for a semi-infinite homogeneous slab $R\left(E, \omega \mid E_{0}, \omega_{0} ; \infty\right)$ is defined as an expected current density of photons per unit area back scattered by the semi-infinite slab with energy $E$ in the direction $\omega$ due to photons of the unit current density per unit area incident uniformly upon the surface of the slab with energy $E_{0}$ in the direction $\omega_{0}$. It is assumed that the incident photons have a rotational symmetry with respect to the $\mathrm{x}$-direction perpendicular to the surface of the slab and the direction of photons is specified by the cosine of the angle between the photon direction and the $\mathrm{x}$-direction. The equation of the reflection function was derived according to the principle of invariance stating that the reflection function of the semi-infinite homogeneous slab is invariant against an addition of a slab with the same composition as the semi-infinite slab [4]. Since the function is free from spatial variable, the equation can be solved more easily than the Boltzmann equation.

\subsubsection{Calculation of modified transmission function}

Suppose we have a plane source located on the surface of the semi-infinite homogeneous slab and that a homogeneous slab of thickness $X$ with the composition same as the semi-infinite slab is placed in front of the plane source as shown in Figure 1.

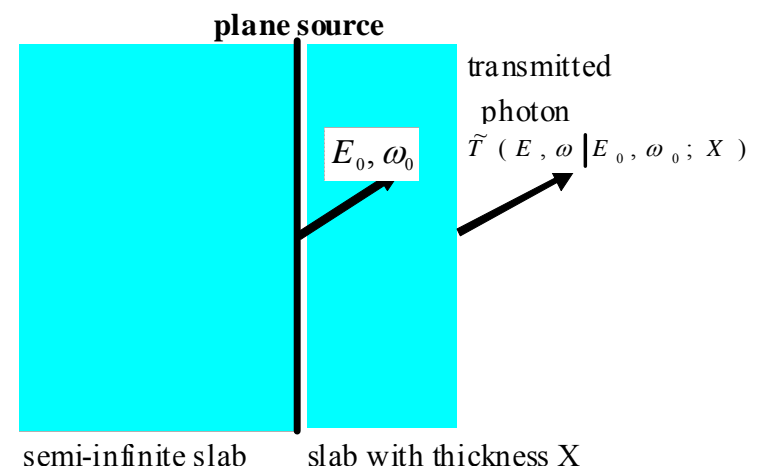

Figure 1. Configuration in defining the modified transmission function.

A modified transmission function of the slab with thickness $X, \widetilde{T}\left(E, \omega \mid E_{0}, \omega_{0} ; X\right)$, is defined as the current density of photons per unit area emerging from the front surface of the slab of the thickness $X$ with energy $E$ in the direction $\omega$ due to the unit current density of photons per unit area emitted from the plane source with energy $E_{0}$ in the forward direction $\omega_{0}\left(\omega_{0} \geq 0\right)$.

The equation of the modified transmission function can be derived by considering how it changes with a change

of the slab thickness by an infinitesimal amount $d X[3]$, given by

$$
\frac{d}{d X} \tilde{T}\left(E, \omega \mid E_{0}, \omega_{0} ; X\right)=-\frac{\Sigma(E)}{\omega} \tilde{T}\left(E, \omega \mid E_{0}, \omega_{0} ; X\right)
$$

$$
+\int_{0}^{E_{0}} d E^{\prime} \int_{0}^{1} d \omega^{\prime} C\left(E, \omega \mid E^{\prime}, \omega^{\prime}\right) \widetilde{T}\left(E^{\prime}, \omega^{\prime} \mid E_{0}, \omega_{0} ; X\right)
$$

where,

$$
\begin{aligned}
& C\left(E, \omega \mid E_{0}, \omega_{0}\right)=\frac{1}{\omega_{0}} \Sigma_{s}\left(E_{0}, \omega_{0} \rightarrow E, \omega\right) \\
& +\int_{0}^{E_{0}} d E^{\prime} \int_{0}^{1} d \omega^{\prime} R\left(E, \omega \mid E^{\prime}, \omega^{\prime} ; \infty\right) \Sigma_{s}\left(E_{0}, \omega_{0} \rightarrow E^{\prime}, \omega^{\prime}\right)(2)
\end{aligned}
$$

and $\Sigma(E)$ is the macroscopic total cross section of the medium, and $\Sigma_{s}\left(E_{0}, \omega_{0} \rightarrow E, \omega\right)$ is the differential cross section for scattering from energy $E_{0}$ to $E$ and from the direction $\omega_{0}$ to $\omega$. The functional equation can also be derived [3], given by

$$
\begin{aligned}
& \widetilde{T}\left(E, \omega \mid E_{0}, \omega_{0} ; X+X^{\prime}\right) \\
& =\int_{0}^{E_{0}} d E^{\prime} \int_{0}^{1} d \omega^{\prime} \tilde{T}\left(E, \omega \mid E^{\prime}, \omega^{\prime} ; X^{\prime}\right) \widetilde{T}\left(E^{\prime}, \omega^{\prime} \mid E_{0}, \omega_{0} ; X\right)
\end{aligned}
$$

\subsubsection{Specific feature of IE method}

The specific features of the IE method as compared with the methods to solve the Boltzmann equation (Boltzmann approach) are as follows :

(1) In the Boltzmann approach, photon flux at all points (practically at a finite number of points) in the slab should be computed simultaneously subject to the boundary conditions given at both slab boundaries (boundary value problem), whereas the transmission function for increasing thickness can be computed successively in the IE method (initial value problem). Initial value problems require less memory and time in computer than boundary value problems.

(2) Since the attenuation of photons with energy $E$ in the direction $\omega$ without any collision with the material is given as $\exp (-\Sigma(E) X / \omega)$, a spatial mesh width $\Delta X \leq \omega / \Sigma(E)$ is required in the spatial integration of Eq.1. The required width is given as $\Delta X \leq \omega /\left(\Sigma(E) / \Sigma\left(E_{0}\right)\right)$ in terms of the mean free path of photons with the source energy $E_{0}$. The required width becomes very small, when the photon direction $\omega$ approaches 0 , and the factor $\Sigma(E) / \Sigma\left(E_{0}\right)$ becomes large for high- $Z$ materials at low energy $E$. The same problem of very small spatial mesh width is met in the Boltzmann approach for photons as well.

The problem is overcome by the IE method. A very small initial mesh $\Delta X$,i.e., $\Delta X=1 / 512 \mathrm{mfp}$ for low-Z materials, and $\Delta X=1 / 2048$ for high-Z materials, is chosen. The Runge-Kutta method is used to the spatial integration of Eq.(1) by the initial mesh $\Delta X$. Solutions for extended thicknesses are obtained successively by use of the functional equation (3). An extension to a slab of large thickness, i.e., $X=100 \mathrm{mfp}$, is easy. An error in solutions due to the spatial integration is confirmed negligibly small (see the subsection 4.4.1).

\subsubsection{Calculation of buildup factors}

Calculations of buildup factors of gamma-rays were made by Shimizu et. al. [5-7] by the IE method. The 
following corrections were made in addition to calculations of the reflection and the modified transmission functions :

(1) The unit current density of source photons emitted with energy $E_{0}$ to the backward direction $\omega_{0}$ from the plane source is equivalent to $R\left(E, \omega \mid E_{0}, \omega_{0} ; \infty\right)$ emitted to the forward direction from the plane source.

(2) A semi-infinite homogeneous medium should be added in front of the surface of the slab of thickness $X$ in Fig. 1 to make an infinite homogeneous medium where the buildup factors are defined. A correction with the effect of the semi-infinite medium placed in front of the slab upon the current density of transmitted photons can be made by using the reflection function $R\left(E, \omega \mid E_{0}, \omega_{0} ; \infty\right)$ of the semi-infinite medium.

(3) A conversion of photon flux for a plane isotropic source in the infinite homogeneous medium $\phi^{p l}(E, X)$ to the corresponding photon flux for a point isotropic source $\phi^{p t}(E, X)$ can be made by the equation

$$
\phi^{p t}(E, X)=-\frac{1}{2 \pi X} \frac{d}{d X} \phi^{p l}(E, X)
$$

\section{Evaluation of error in numerical solutions}

\subsection{Introduction}

An extensive work has been made to evaluate the magnitude of error in buildup factors obtained by the IE method. It includes : 1) comparison with the ANS standard data obtained by the moments method, 2) comparison with EGS4 calculations, and 3) self estimation of error in numerical solutions by analyzing influence of mesh width in space, energy and angle on buildup factors.

\subsection{Comparison with the moments method}

Gamma-ray buildup factors for 19 low-Z materials including water, concrete and air in the ANS data were generated by the moments method. The method has an excellent feature that an accurate solution is obtainable for the spatial moments, although a reconstruction of the flux density from the spatial moments sometimes results in spurious oscillation [2]. Extensive calculations of exposure buildup factors for 19 low-Z materials and 25 source energies (475 cases) were made by the IE method for comparison based on the cross section NBS29 [8].

As bremsstrahlung was ignored in moment calculation, bremsstrahlung was ignored in IE calculation to make condition the same. The result of comparison is as follows : Both data coincide up to $40 \mathrm{mfp}$ with discrepancy less than $5 \%$ for 391 cases $(82 \%)$ out of 475 cases, and with discrepancy less than $10 \%$ for 453 cases $(391+61$ cases $)(95 \%)$. A discrepancy more than $10 \%$ is observed for 22 cases $(5 \%)$ including $\mathrm{Be} 0.02 \mathrm{MeV}$ and others. Based on the self estimation of error in the IE method described in the subsection 4.4, the discrepancy in these 22 cases is found due to spurious oscillation in the moments method.

\subsection{Comparison with EGS4 calculations}

Exposure buildup factors were calculated by using Monte Carlo code EGS4 by Hirayama for water, iron and lead with the source energies $0.1,1.0$ and $10 \mathrm{MeV}$ [9]. Calculations for comparison were made by the IE method with the same cross section and the bremsstrahlung model as used in the EGS4 calculations. The result of comparison is summarized in Table 1. It is shown that buildup factors by both methods coincide with a discrepancy of about $10 \%$ or less, except for a discrepancy of $15 \%$ observed for lead with $10 \mathrm{MeV}$ at 30-40 mfp.

Table 1. Ratio of exposure buildup factor obtained by EGS4 to that obtained by IE method.

\begin{tabular}{|c|c|c|c|c|c|c|c|c|}
\hline$/ \mathrm{X}(\mathrm{mfp})$ & 1 & 5 & 10 & 15 & 20 & 30 & 35 & 40 \\
\hline water $0.1 \mathrm{MeV}$ & 1.02 & 1.06 & 1.08 & 1.09 & 1.05 & 1.03 & 1.01 & 1.05 \\
\hline iron $0.1 \mathrm{MeV}$ & 1.00 & 1.01 & 1.02 & 1.02 & 1.03 & 1.04 & 1.05 & 1.04 \\
\hline lead $0.1 \mathrm{MeV}$ & 1.01 & 1.02 & 1.03 & 1.02 & 1.01 & 0.99 & 0.99 & 0.98 \\
\hline water $1 \mathrm{MeV}$ & 1.01 & 1.02 & 1.01 & 1.04 & 1.01 & 1.01 & 1.01 & 0.99 \\
\hline iron $1 \mathrm{MeV}$ & 1.00 & 1.00 & 1.01 & 1.03 & 1.00 & 1.02 & 1.03 & 1.05 \\
\hline lead $1 \mathrm{MeV}$ & 1.01 & 1.02 & 1.03 & 1.02 & 1.03 & 1.04 & 1.06 & 1.09 \\
\hline water $10 \mathrm{MeV}$ & 1.01 & 1.01 & 1.02 & 1.03 & 1.02 & 1.05 & 1.05 & 1.05 \\
\hline iron $10 \mathrm{MeV}$ & 1.00 & 1.01 & 1.01 & 1.03 & 1.06 & 1.07 & 1.09 & 1.08 \\
\hline lead $10 \mathrm{MeV}$ & 1.00 & 1.00 & 1.02 & 1.03 & 1.04 & 1.08 & 1.10 & 1.13 \\
\hline lead-B* $10 \mathrm{MeV}$ & 1.01 & 1.04 & 1.05 & 1.08 & 1.09 & 1.13 & 1.15 & 1.11 \\
\hline
\end{tabular}

\subsection{Self estimation of error in numerical solutions}

\subsubsection{Influence of spatial mesh width}

According to the IE method, Eq. (1) is integrated numerically at an initial spatial mesh width $\Delta X$ by the Lung-Kutta method. An error in the solution is estimated to be between $\Delta X^{4}$ and $\Delta X^{5}$. When an initial mesh width is reduced to $\Delta X / 2$, an error of the solution at the thickness $\Delta X$, that is obtained by using the solution at the initial thickness $\Delta X / 2$ and by using once the functional equation (3), is estimated between $2(\Delta X / 2)^{4}$ and $2(\Delta X / 2)^{5}$, taking the effect of the functional equation (3) upon the error of the solution. It is confirmed numerically for water at the source energy of $1.0 \mathrm{MeV}$ shown in Table 2.

It is indicated that an error of the solution is reduced to about $1 / 10$, when the initial mesh width is reduced to $1 / 2$. The standard data of buildup factors are calculated by the IE method with the initial mesh width of $1 / 512$ $\mathrm{mfp}$ for low-Z materials, $1 / 2048 \mathrm{mfp}$ for high-Z materials, and $1 / 8192 \mathrm{mfp}$ for calculations with bremsstrahlung. The magnitude of error in the data is confirmed less than $0.1 \%$ up to depth of $100 \mathrm{mfp}$ through check calculations with the initial mesh width reduced to $1 / 2$.

\subsubsection{Influence of angular and energy mesh width}

Numerical calculations of the standard buildup factors were made by the IE method bases on the discrete 
Table 2. Influence of an initial mesh width upon buildup factors obtained by IE method Water $1.0 \mathrm{MeV}$.

\begin{tabular}{ccccc}
\hline \multicolumn{5}{c}{$\mathrm{BF}(\Delta) / \mathrm{BF}(\Delta \mathrm{min}) \Delta \mathrm{min}=1 / 1024 \mathrm{mfp}$} \\
\hline $\begin{array}{c}\mathrm{X} / \Delta \\
(\mathrm{mfp})\end{array}$ & $1 / 64 \mathrm{mfp}$ & $1 / 128 \mathrm{mfp}$ & $1 / 256 \mathrm{mfp}$ & $1 / 512 \mathrm{mfp}$ \\
\hline 1 & & & & \\
10 & 1.0967 & 1.0119 & 1.0010 & 1.0000 \\
20 & 1.2328 & 1.0324 & 1.0030 & 1.0002 \\
40 & 1.2458 & 1.0344 & 1.0032 & 1.0002 \\
100 & 1.2525 & 1.0355 & 1.0033 & 1.0002 \\
\hline
\end{tabular}

ordinate approximation with 15 divisions of the angular variable in the range $0 \leq \omega \leq 1$ and the multi-group approximation with 95 groups of energy in the range between the source energy and a cutoff energy. Survey calculations are performed to evaluate the magnitude of error in buildup factors as functions of the number of angular divisions and energy groups. We denote an exposure buildup factor calculated with a number of angular divisions $\mathrm{G}$ and a number of energy groups $\mathrm{N}$ at a depth $\mathrm{X}$ by $\mathrm{BF}(\mathrm{G}, \mathrm{N} ; \mathrm{X})$. It is found that $\mathrm{BF}(\mathrm{G}, \mathrm{N} ; \mathrm{X})$ tends to increase with $G$ increasing. The ratio $\mathrm{BF}(15,95 ; \mathrm{X}) / \mathrm{BF}(13,95 ; \mathrm{X})$ for water, iron and lead is shown in Table 3.

Table 3. Ratio of buildup factor computed by 15 divisions to that by 13 divisions in angle $: \mathrm{BF}(15,95 ; \mathrm{X}) / \mathrm{BF}(13,95 ; \mathrm{X})$

\begin{tabular}{lcccc}
\hline M aterial & $\begin{array}{c}\mathrm{X}(\mathrm{mfp}) \\
\mathrm{E}_{0}(\mathrm{MeV})\end{array}$ & 10 & 40 & 100 \\
\hline Water & 0.1 & 1.000 & 1.003 & 1.009 \\
& 1.0 & 1.001 & 1.007 & 1.018 \\
& 10.0 & 1.003 & 1.006 & $(1.001)^{*}$ \\
\hline Iron & 0.1 & 1.000 & 1.002 & 1.006 \\
& 1.0 & 1.000 & 1.002 & 1.006 \\
& 10.0 & 1.003 & 1.006 & $(1.000)^{*}$ \\
\hline Lead & 0.1 & 1.000 & 1.000 & 1.000 \\
& 1.0 & 1.001 & 1.004 & 1.011 \\
& 10.0 & 1.001 & 1.007 & 1.020 \\
\hline * condition for extrapolation is not satisfied &
\end{tabular}

Table 4. Ratio of buildup factor computed by 95 groups to that by 85 groups in energy : $\mathrm{BF}(15,95 ; \mathrm{X}) / \mathrm{BF}(15,85 ; \mathrm{X})$

\begin{tabular}{lcccc}
\hline & $\begin{array}{c}/ \mathrm{X}(\mathrm{mfp}) \\
\text { M aterial }\end{array}$ & 10.0 & 40.0 & 100.0 \\
\hline Water & 0.1 & 1.000 & 0.999 & 0.993 \\
& 1.0 & 0.998 & 0.995 & 0.979 \\
& 10.0 & 0.999 & 0.997 & 0.986 \\
\hline Iron & 0.1 & 1.000 & 0.997 & 0.982 \\
& 1.0 & 1.000 & 0.999 & 0.995 \\
& 10.0 & 0.997 & 0.997 & 0.995 \\
\hline Lead & 0.2 & 1.000 & 0.998 & 0.992 \\
& 1.0 & 1.000 & 0.995 & 0.973 \\
& 10.0 & 1.002 & 1.001 & 1.000 \\
\hline
\end{tabular}

It is also found that buildup factors tend to decrease with $\mathrm{N}$ increasing, as shown in Table 4.

According to the method of extrapolation introduced by Shimizu [7], the upper limit of $B F(G, N ; X)$ when $G$ goes to $\infty$, and the lower limit of $B F(G, N ; X)$ when $N$ goes to $\infty$ are obtainable. Based on the table 3 and 4 , these limits are given as :

$$
\begin{array}{ll}
\text { at } X=40 \mathrm{mfp}, & \mathrm{BF}(\infty, 95 ; \mathrm{X}) \geq 1.03 \mathrm{BF}(15,95 ; \mathrm{X}), \\
& \mathrm{BF}(15, \infty ; \mathrm{X}) \leq 0.95 \mathrm{BF}(15,95 ; \mathrm{X}), \\
\text { at } \mathrm{X}=100 \mathrm{mfp} \quad \mathrm{BF}(\infty, 95 ; \mathrm{X}) \geq 1.07 \mathrm{BF}(15,95 ; \mathrm{X}), \\
& \mathrm{BF}(15, \infty ; \mathrm{X}) \leq 0.80 \mathrm{BF}(15,95 ; \mathrm{X}) .
\end{array}
$$

\subsection{Conclusion}

The magnitude of error in buildup factors obtained by the IE method is about $10 \%$ or less up to the depth of 40 mfp, and will be about $20 \%$ or less up to depth of 100 $\mathrm{mfp}$. The error due to the spatial integration is confirmed negligible (less than $0.1 \%$ ) up to depth of $100 \mathrm{mfp}$.

\section{References}

[1] Y. Sakamoto, H. Hirayama, O. Sato and A. Shimizu, Generation and application of bremsstrahlung production data calculated EGS4 code, Proc. ICRS-11, April 13-18, 2008, Georgia, USA, (2008).

[2]American National Standard, Gamma-Ray Attenuation Coefficients and Buildup Factors for Engineering Materials, ANSI/ANS-6.4.3, (1991).

[3] Radiation Shielding Information Center Data Package DLC-136/PHOTX, Photon Interpolation Cross Section Library, National Institute of Standards and Technology.

[4] A. Shimizu and K. Aoki, Application of Invariant Embedding to Reactor Physics, Academic Press, (1972).

[5] A. Shimizu, Calculation of gamma-ray buildup factors up to depths of $100 \mathrm{mfp}$ by the method of invariant embedding, (I), J. Nucl. Sci. Technol., 39 (2002), pp. 477-486.

[6] A. Shimizu and H. Hirayama, Calculation of gamma-ray buildup factors up to depths of $100 \mathrm{mfp}$ by the method of invariant embedding, (II), J. Nucl. Sci. Technol., 40 (2003), pp. 192-200.

[7] A. Shimizu, T. Onda and Y. Sakamoto, Calculation of gamma-ray buildup factors up to depths of 100 mfp by the method of invariant embedding, (III), J. Nucl. Sci. Technol., 41 (2004), pp. 413-424.

[8] J. H. Hubell, Photon Cross Sections, Attenuation Coefficients, and Energy Absorption Coefficienta From $10 \mathrm{keV}$ to $10 \mathrm{GeV}$, NSRDS-NBS29, National Bureau of Standards (1969).

[9] H. Hirayama, Calculation of gamma-ray exposure buildup factors up to $40 \mathrm{mfp}$ using the EGS4 Monte Carlo code with a particle splitting, J. Nucl. Sci. Technol., 32 (1995), pp. 1201-1207. 